\title{
Inexpensive optically-isolated nanoammeter for use with micro-Newton electric propulsion technology
}

\author{
Karen L. Aplin ${ }^{\mathrm{a}}$ \\ Rutherford Appleton Laboratory, Didcot, Oxon, OX11 0QX, UK \\ Katharine L. Smith ${ }^{\mathrm{b}}$ \\ Queen Mary, University of London, London, E1 4NS, UK \\ John G. Firth ${ }^{\text {c }}$, Barry J. Kent ${ }^{\mathrm{d}}$ \\ Rutherford Appleton Laboratory, Didcot, Oxon, OX11 0QX, UK \\ Matthew S. Alexander ${ }^{\mathrm{e}}$ and John P.W. Stark ${ }^{\mathrm{f}}$ \\ Queen Mary, University of London, London, E1 4NS, UK
}

\section{Nomenclature}

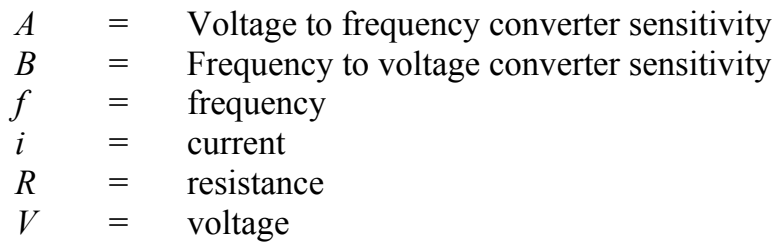

\section{Introduction}

The demanding scientific requirements of several future space missions require accurate station keeping. These include fundamental physics experiments needing drag free control such as LISA and LISA Pathfinder, and formation flying astronomy missions like DARWIN and XEUS. This has motivated research into micro-Newton electric propulsion technologies, for example colloidal thrusters and field emission electric propulsion (FEEP) ${ }^{1}$. In the development of these thrusters, small (nanoAmpere) emission currents often need to be measured and logged when the thruster itself is held at kilovolt potentials with respect to ground. The need for safe, yet precise measurement of small currents at high voltage presents a technical challenge, particularly for colloidal thruster development where the emitter is held at $10 \mathrm{kV}$. Previous work on colloid thruster systems has circumvented these

\footnotetext{
${ }^{a}$ Research Physicist, Space Science and Technology Department, Rutherford Appleton Laboratory, Chilton, Didcot, Oxon OX11 0QX and AIAA non member. (email: k.l.aplin@rl.ac.uk)

${ }^{\mathrm{b}}$ Postdoctoral Researcher, Department of Engineering, Queen Mary, University of London, London E1 4NS and AIAA non member.

${ }^{\mathrm{c}}$ Electronics Engineer, Space Science and Technology Department, Rutherford Appleton Laboratory, Chilton, Didcot, Oxon OX11 0QX (now retired) and AIAA non member.

${ }^{\mathrm{d}}$ Applied Physics and Systems Engineering Group Leader, Space Science and Technology Department, Rutherford Appleton Laboratory, Chilton, Didcot, Oxon OX11 0QX (now an independent space consultant) and AIAA non member.

${ }^{\mathrm{e}}$ Lecturer, Department of Engineering, Queen Mary, University of London, London E1 4NS and AIAA non member.

${ }^{\mathrm{f}}$ Head of Department, Department of Engineering, Queen Mary, University of London, London E1 4NS and AIAA non member.
} 
K.L. Aplin et al

J. Propulsion Power (2008) doi: 10.2514/1.30994.2514/1.30994

difficulties by placing a flat plate collector, nominally at ground potential, in the spray path, and measuring the resulting current. However measuring the current in this manner can produce erroneous results if the entire spray is not collected on the plate. In a vacuum system, surrounding objects may attract some of the spray and reduce the collector efficiency. An alternative approach is to measure the emitted colloid spray current. This gives a more accurate value of thruster current, as the total electrospray current emitted is not susceptible to spray collection errors. Here we report the design and implementation of an inexpensive optically isolated nanoammeter which permits digital logging of dc currents measured at $\sim 10 \mathrm{kV}$ from an electrospray colloid thruster operated in vacuum ${ }^{2}$. Two of these nanoammeters are used to detect the emitter current and also the losses to the accelerating grid electrode, or extractor.

\section{Overview}

The two-stage optically isolated system is intended to safely measure and record a current generated at a high voltage, facilitating data acquisition with a grounded logging device such as a personal computer. It operates by converting the current into a frequency in a battery-powered, floated, highvoltage stage. The frequency is transmitted optically to a frequency-to-voltage converter, operated at the local potential of the data acquisition system. Similar circuits described in the literature include an opto-coupled current mirror ${ }^{3}$, which would be inappropriate for this application since minimum currents $\sim 100 \mu \mathrm{A}$. Another approach is to pass the current to be measured through a light emitting diode, which is detected using a photodiode. Current-to-voltage conversion takes place in the lowpotential part of the circuit ${ }^{4,5}$. Transmission of a photocurrent, rather than a frequency, could be susceptible to losses and temperature-dependent errors. The systems in [3] and [4] were only designed to operate up to $3.5 \mathrm{kV}$, so additional safety precautions are required in the $10 \mathrm{kV}$ colloidal thruster system. Ref. [5] reports results obtained at $10 \mathrm{kV}$, but here we have chosen to implement some of the safety precautions described in an optically-isolated voltmeter follower for use at potentials of up to $10 \mathrm{kV}^{6}$

Agilent Technologies fibre optic kits (part number HFBR-0501) were selected for their low cost and reliability. The basic system (used in this paper) permits transmission over $120 \mathrm{~m}$ at $40 \mathrm{kBd}$, and is readily modified for high speed transmission at $5 \mathrm{MBd}$. 


\section{A. System description}

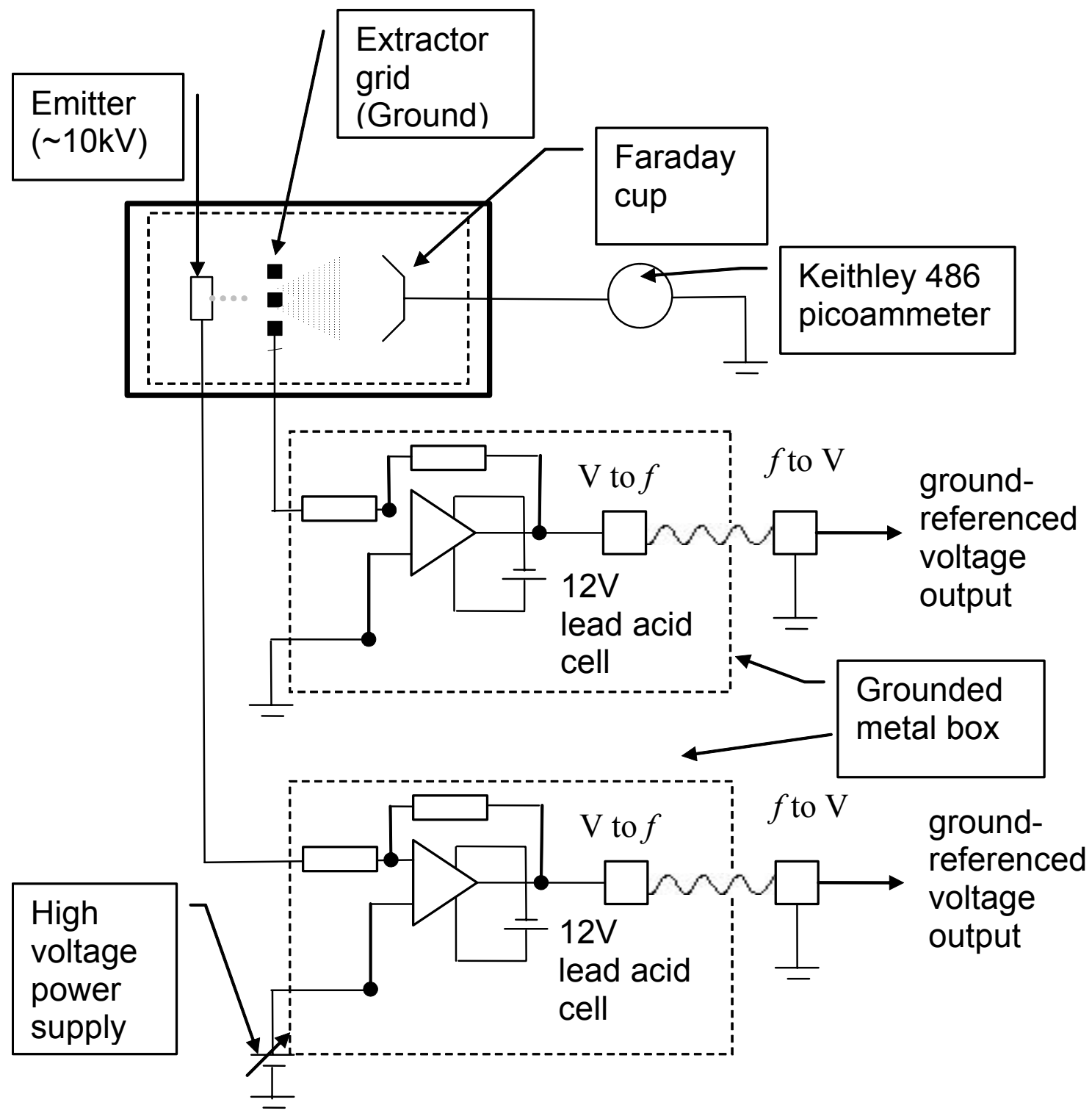

Figure 1 Overview of the system. The thick box denotes the vacuum tank housing the thruster test apparatus (internal vacuum $\sim 10^{-5}$ torr), and dashed lines indicate the high voltage sections. Both the emitter and extractor currents are measured, using two optically-isolated systems (this allows either to be operated at high voltage). The spray current collected by an unbiased Faraday cup is also measured using a Keithley 486 picoammeter.

A schematic diagram of the system is given in Figure 1. A battery-powered high voltage stage, floated at the emitter and extractor electrode potential, contains a current to voltage (i-V) converter followed by a voltage to frequency (V-f) converter. After optical transmission to a grounded $\mathrm{f}-\mathrm{V}$ converter, the system outputs a ground-referenced unipolar voltage that is linearly proportional to the electrospray current. Safety features have been implemented using microswitches to cut out the high voltage if the 
K.L. Aplin et al

J. Propulsion Power (2008) doi: 10.2514/1.30994.2514/1.30994

circuit enclosure is disturbed. The spray current, which provides the thrust, is also detected by measuring the current to an unbiased Faraday cup collector with a Keithley 486 picoammeter.

\section{Circuit Description}

In the high-voltage transmitter part of the circuit, Figure 2a, a transresistance $\mathrm{i}-\mathrm{V}$ converter, with a precision temperature-stable feedback resistor of $1 \mathrm{M} \Omega \pm 1 \%$ measures currents to $\pm 5 \mu \mathrm{A}$ with a sensitivity of $\sim-1 \mathrm{mV} / \mathrm{nA}$. The output voltage is converted to frequency using an AD650 voltage to frequency converter chip, chosen for its high linearity. The AD650 can also be used for the frequency to voltage converter in the grounded receiving stage (Figure 2b). In the transmitter, the AD650 gives a full scale frequency output of $50-150 \mathrm{kHz}$, and the fibre optic output is driven by an SN75451 optical driver chip. The circuit is powered by two Yuasa 1.2Ah $12 \mathrm{~V}$ sealed lead acid batteries, from which an LM2931 5V voltage regulator supplies the SN75451.

The circuitry and batteries are mounted on a circuit board placed on Paxolin spacers and secured with insulating nylon screws to a grounded metal box. The components were sufficiently spaced from the box to withstand voltages $\sim 100 \mathrm{kV}$, based on a breakdown field of $30 \mathrm{kVcm}^{-1}$ for air. This is well above the maximum output voltage of the high voltage supplies $(\sim 10 \mathrm{kV})$ used with the experimental system. A simulated roller microswitch (Saia-Burgess 5A) is positioned on the rim of the box and connected to the high voltage supply safety interlock. This disconnects the voltage supply if the lid is removed from the box. A $15 \mathrm{M} \Omega$ temperature stable high-voltage series resistor at the input protects the op-amp from transients. Even at high voltage the maximum voltage drop across this resistor is negligible.

The fibreoptic cable passes between the high voltage and the grounded receiver stage via grommeted holes drilled in the metal housing. The grounded receiver stage (Figure 1b) uses the AD650 in $f-V$ configuration to convert the $50-150 \mathrm{kHz}$ signal into a voltage centred at $5 \mathrm{~V}$. It requires a $\pm 12 \mathrm{~V}$ power supply for a positive single-ended signal. Another SN75451 chip running from a $5 \mathrm{~V}$ regulator is used to drive the optical receiver. 


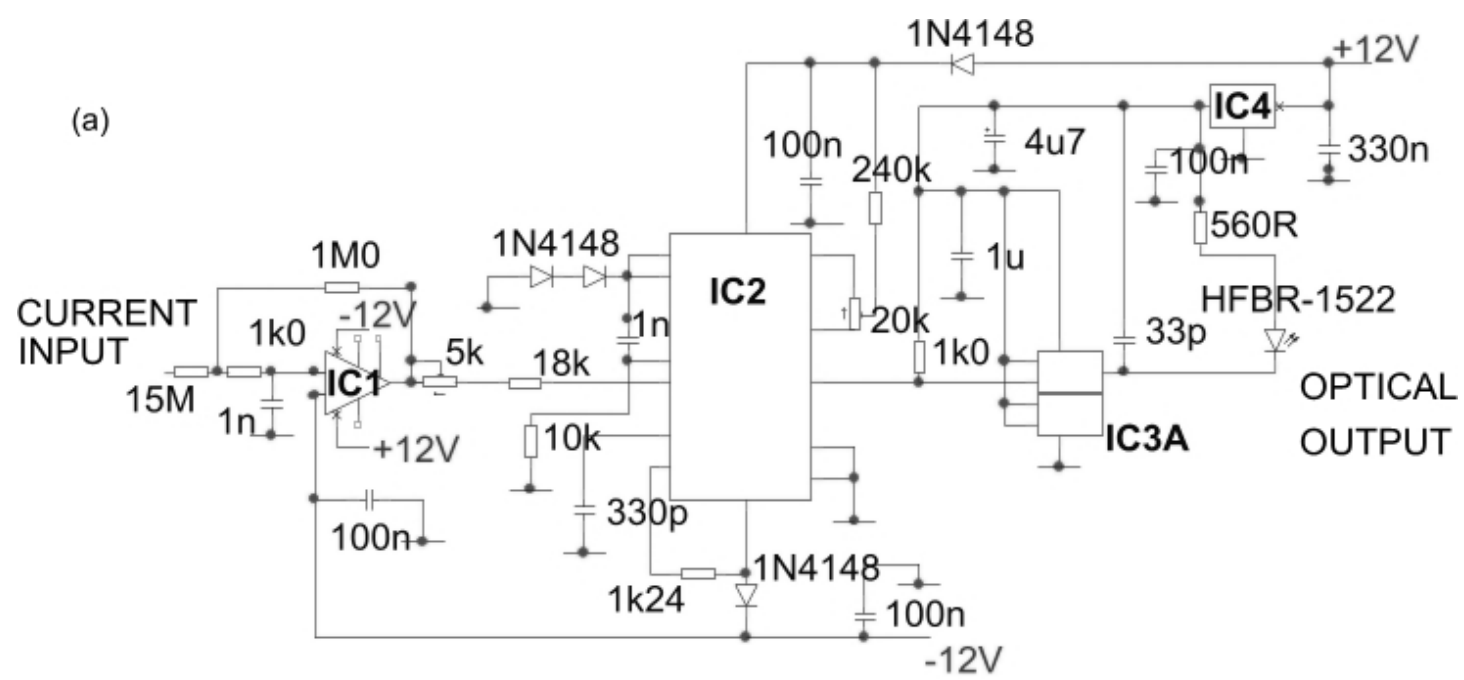

(b)

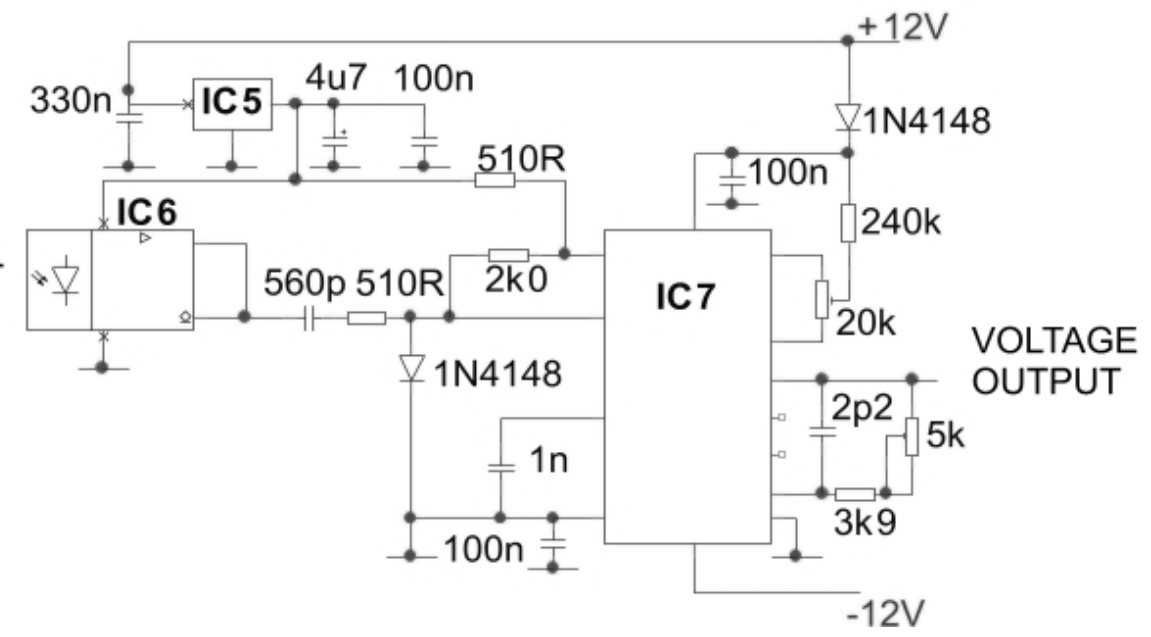

Figure 2 Schematic diagram of the opto-isolated current measurement system (a) Current to frequency converter, floated at high tension. IC1 OP97 operational amplifier, IC2 AD650JN in voltage to frequency converter configuration, IC3A SN745451BP optical driver, IC4 LM2931AZ5 5V regulator, optical output HFBR-2522 fibre optic transmitter. $\pm 12 \mathrm{~V}$ is supplied from 2xYuasa 1.2Ah 12V sealed lead acid batteries (b) frequency to voltage converter. IC5 LM2931 AZ-5 optical driver, IC6 HFBR2522 fibre optic receiver, IC7 AD650JN in frequency to voltage converter configuration. 
K.L. Aplin et al

J. Propulsion Power (2008) doi: 10.2514/1.30994.2514/1.30994

\section{System Characterisation}

The response of the system to a current $i$ is given by

$$
\mathrm{V}_{\text {out }}=\mathrm{AB} i \mathrm{R}
$$

where $\mathrm{R}$ is the $\mathrm{i}-\mathrm{V}$ converter feedback resistor $(1 \mathrm{M} \Omega \pm 1 \%)$, $\mathrm{A}$ is the $\mathrm{V}$-f converter sensitivity and $\mathrm{B}$ is the $\mathrm{f}-\mathrm{V}$ converter sensitivity. The $\mathrm{i}-\mathrm{V}$ converter was calibrated separately by resistively generating a current using a Time Electronics 2003 millivolt calibrator across a $1 \mathrm{M} \Omega \pm 1 \%$ resistor, and measuring the voltage output using a Thandar 1905a precision digital multimeter (DMM). A was found to be $18.64 \pm 0.05 \mathrm{~V} / \mathrm{kHz}$ by generating a frequency using a Lyons PG71N bipolar pulse generator and measuring the output voltage with a DMM. Using a similar approach, B was determined as $0.0241 \pm 0.0000 \mathrm{kHz} / \mathrm{V}$ by using the millivolt calibrator to generate an input voltage, and measuring the frequency output with a Gould Classic 6000 digital storage oscilloscope. As $\mathrm{R}=1 \mathrm{M} \Omega \pm 1 \%$.. then the system response is predicted to be $(0.4 \mathrm{mV} / \mathrm{nA}) \pm 1 \%$. These results are shown in Figure 3a-c.

The complete system can be calibrated by generating a current resistively as for the $\mathrm{i}-\mathrm{V}$ converter calibration, and measuring the output voltage with a DMM. During calibration, the input stage is not floated, and the calibration current is applied after the $15 \mathrm{M} \Omega$ input protection resistor. The system calibration is shown in Figure 3d. 


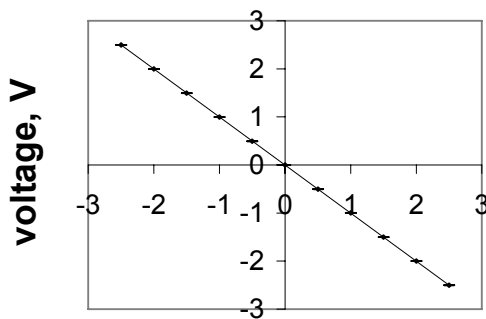

(a)

current, uA

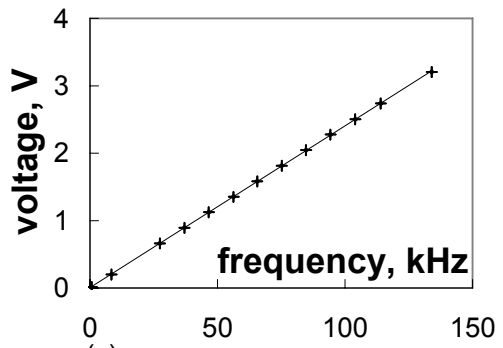

(c)
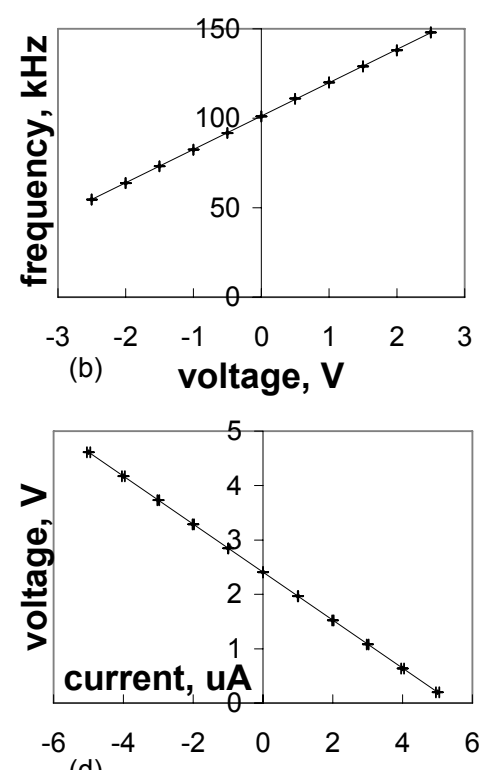

(d)

Figure 3 Calibration of the system. Crosses over the points represent the errors in the measurements. Linear regression lines are also shown. (a) i-V converter calibration, response $0.9989 \pm 0.0003 \mathrm{mV} / \mathrm{nA}$, (b) $\mathrm{V}$-f converter calibration, response $18.64 \pm 0.05 \mathrm{~V} / \mathrm{kHz}$ (c) f-V converter calibration, response $0.02405 \pm 0.00003 \mathrm{kHz} / \mathrm{V}$ (d) calibration of the complete system showing output voltage against resistively generated input current, response $=\mathbf{- 0 . 4 4 1 7 0 \pm 0 . 0 0 0 0 3 ~} \mathrm{V} / \mu \mathrm{A}$, with a zero offset of $2.4069 \pm 0.0001 \mathrm{~V}$.

As Figure 3 indicates, the basic system response is inverting because of the $i-V$ converter, but this can readily be reversed by swapping the inputs as in Figure 1a. The principal error in the calibration results from the $1 \%$ tolerance of the $1 \mathrm{M} \Omega$ resistor used to generate the calibration current (the $\mathrm{mV}$ calibrator tolerance is $\pm 0.05 \%$, and linear regression errors $<<1 \%$ ). The system response is measured as $(0.4 \mathrm{mV} / \mathrm{nA}) \pm 0.01 \%$, and the lowest measurable current is $\sim 10 \mathrm{nA}$. A small additional error is introduced during operation at high voltage, from the voltage drop across the protection resistor generated by the emitter current. At the maximum currents expected, $5 \mu \mathrm{A}$, the voltage drop will be $75 \mathrm{~V}$ giving a maximum error of $0.075 \%$, which is an order of magnitude smaller than the errors in the calibration. After $\sim 4$ years deployment in a colloidal thruster development rig, a recent recalibration indicated a drift in the response of $4 \%$ with no loss of linearity. Annual recalibration is therefore recommended to keep the system capable of measuring current to $\pm 1 \%$. 
K.L. Aplin et al

J. Propulsion Power (2008) doi: 10.2514/1.30994.2514/1.30994

In an additional characterization experiment, the response time of the system to a change in current (generated by switching a voltage from a Time Electronics $2003 \mathrm{~S}( \pm 0.02 \%)$ millivolt calibrator through a $100 \mathrm{k} \Omega \pm 0.1 \%$ resistor) was measured using a Wavestar 424 oscilloscope sampling at 10ksamples/s. The calibrated response of the optically isolated system to the change in current is shown in Figure 4. The input pulse took $17 \mathrm{~ms}$ from the zero time point (nominally triggered in the middle of the pulse) to reach $95 \%$ of its final value. The isolated current measurement system took $20 \mathrm{~ms}$ to reach $95 \%$ of its final value, therefore the time response of the system is estimated to be $3 \mathrm{~ms}$. The overshoot was $\sim 25 \%$.

(a)

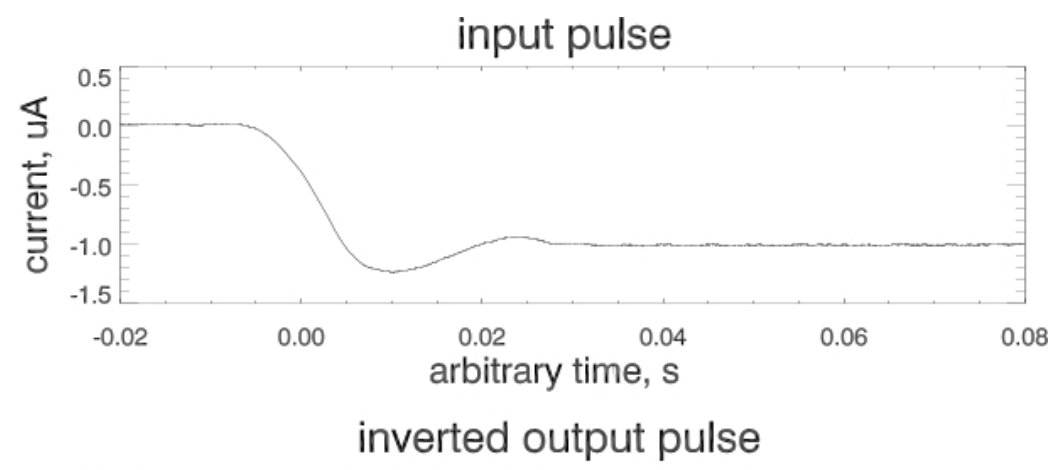

(b)

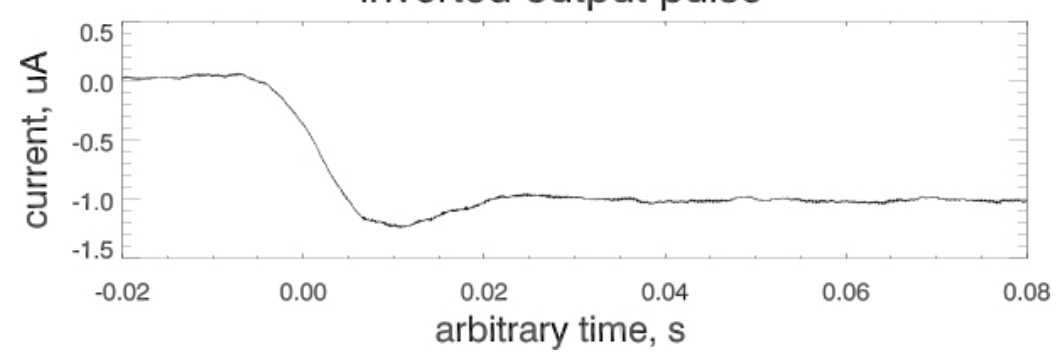

Figure 4 Test of system time response, showing $100 \mathrm{~Hz}$ centred moving averages of $10 \mathrm{kHz}$ sampled data (a) input current pulse, generated by switching a millivolt calibrator through a precision resistor. (b) system response.

The optically isolated system introduces some noise, which can be estimated by comparing the variability of the input signal $(t<-0.005 \mathrm{~s}$ on Figure 4$)$ to the processed output pulse at steady-state $(t>0.04 \mathrm{~s})$. If 100 point $(100 \mathrm{~Hz})$ averages of the sampled signal are used, the input pulse steady-state value was $-1.01 \pm 0.01 \mu \mathrm{A}$ and the corresponding output current was $-1.01 \pm 0.04 \mu \mathrm{A}$, where the errors are the standard deviation. This small increase in noise and the larger overshoot are likely to be negligible in practice, since electric propulsion currents are more commonly sampled at $\sim 1 \mathrm{~Hz}$, when these fluctuations from the circuitry would be averaged out. 
K.L. Aplin et al

J. Propulsion Power (2008) doi: 10.2514/1.30994.2514/1.30994

\section{Operation}

The electrospray emitter was floated to $10 \mathrm{kV}$ above ground using a high voltage power supply. The optically isolated nanoammeter was placed in series with this power supply between the emitter and the high tension side of the power supply, Figure 1. (An alternative arrangement with the extractor grid floated to $-10 \mathrm{kV}$ with respect to ground can also be used.) The nanoammeter output voltage was connected to a National Instruments 6023E data acquisition card, which has a sampling rate of 66.67ksamples/s. A National Instruments LabVIEW program was used to record the time-averaged signal at a frequency of $\sim 0.5 \mathrm{~Hz}$. Examples of the recorded currents for a single gold coated emitter of outer diameter $170 \mu \mathrm{m}$, inner diameter $60 \mu \mathrm{m}$, running at a flow rate of $18 \mathrm{~nL} / \mathrm{s}$ with $12.5 \mathrm{~g} / 1$ sodium iodide in triethylene glycol are shown in Figure 5. The currents measured on the extractor grid, the Faraday cup and the emitter current are plotted, as well as the sum of the grid and Faraday cup currents or the effective emitter current. The discrepancy between the effective and measured emitter currents is due to the positioning of the Faraday cup, which was $7 \mathrm{~cm}$ downstream of the emitter exit. Since the spray is diverging, some of the emitter current was not collected by the Faraday cup. The combination of spray divergence and nanoammeter system errors must be $<2 \%$, the difference between the mean effective emitter current and emitter current. This gives confidence in the reliability and accuracy of the optical-isolated nanoammeter system.

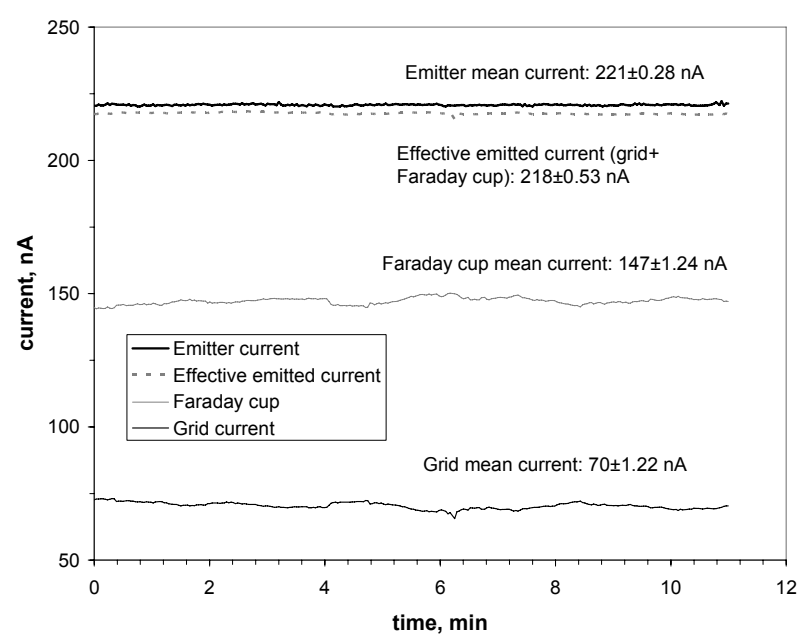

Figure 5 Current measurements for a single integrated nozzle-grid assembly. 
K.L. Aplin et al

J. Propulsion Power (2008) doi: 10.2514/1.30994.2514/1.30994

\section{Discussion}

This optically isolated nanoammeter provides an inexpensive (total parts cost $\sim £ 200 / \$ 400$ ), thermally and temporally stable, and safe way to accurately measure currents generated at $\sim 10 \mathrm{kV}$ in laboratory testing of a colloidal thruster. We are not aware of any similarly isolated commercially available systems, but production costs are an order of magnitude lower than the purchase price of commercially available picoammeters, which are not optically isolated ${ }^{g}$. The low cost of the system permits measurement of both the emitter current and losses to the extractor which can give additional physical insight into the operation of the colloidal thruster system. The tri-electrode (emitter, extractor and spray) current measurement system adopted has allowed easy determination of the presence of any additional currents due to electron emission, or backstreaming of the spray. If these effects are occurring at all, they must be at the $<1 \%$ level due to the close correspondence between the sum of the extractor and spray currents and the emitter current, Figure 5. The system could readily be modified for other applications, for example measurement of the currents from FEEP thrusters. FEEP thrusters are run at $\sim 10 \mathrm{kV}$ and, as for colloidal thrusters, the emission current is rarely measured, since it is more straightforward to detect the current collected at ground potential. However this is subject to error, as was described in Section I. If emission currents are measured, a typical approach is to use the "current monitor" option on the high voltage power supply ${ }^{\mathrm{h}}$. This is clearly non-ideal at low current levels, and a greater physical understanding could be gained by using an isolated current measurement system similar to the one described here.

The system can readily be modified to measure different current ranges by changing the value of the $i$ $V$ converter feedback resistor (in parallel with IC1 in Figure 2), to adjust the gain. With some additional modifications such as careful component choice (e.g. ultra-low input bias current op-amps) and air wiring (where the input pin of the op-amp used is bent upwards and soldered directly to the input to prevent leakage across the board), this system could be straightforwardly adapted to measure currents down to $1 \mathrm{fA}^{7}$. Dedicated femtoammeter calibrators have already been developed to counteract the thermal stability problems that can ensue from resistive generation of very small currents across large resistors for calibration ${ }^{8}$. Increased component spacing within the enclosure and/or an increase in the size of the enclosure would also permit operation at voltages $>10 \mathrm{kV}$. A novel feature is that the use of

\footnotetext{
g www.keithley.com (February 2007)

$\mathrm{h}$ Personal communication, Pierre-Etienne Frigot, European Space Agency
} 
K.L. Aplin et al

J. Propulsion Power (2008) doi: 10.2514/1.30994.2514/1.30994

a fibre-optic cable removes the need for a line-of-sight link between the transmitter and receiver stages, making the system particularly appropriate for confined spaces or limited geometries.

Use of an optically isolated system like this one permits accurate monitoring of micro-Newton thruster currents directly rather than through inaccurate proxies, thus giving physical insight into the behavior of the system at high accuracy and low cost.

\section{Acknowledgments}

This work was funded by the UK Engineering and Physical Sciences Research Council, from whom

KLS acknowledges a studentship.

\section{References}

\footnotetext{
${ }^{1}$ Micci M. and Ketsdever A.D. (eds) "Micropropulsion for small spacecraft", American Institute of Aeronautics and Astronautics, 2001

${ }^{2}$ Alexander M.S., Stark J., Smith K.L., Stevens R. and Kent B.J., "Electrospray performance of microfabricated colloid thruster arrays", Journal of Propulsion and Power, 2006, vol 22 no. 3, pp 620-627

${ }^{3}$ Argiro S., Camin D.V., Destro M. and Guérard C.K., "Monitoring DC anode current of a grounded-cathode photomultiplier tube", Nuclear Instruments and Methods in Physics Research A.,1999, vol 435, pp 484-489

${ }^{4}$ Band A. and Unguris J., "Optically isolated current-to-voltage-converter for an electron optics system", Review of Scientific Instruments, 1998, vol 68, no. 1, pp 250-251

${ }^{5}$ Bailey A.G, Cheung W.L. and Smallwood J. M., "A high-resolution probe for space potential measurements", Journal of Electrostatics, 1995, vol 36, no. 2, pp 151-163

${ }^{6}$ Harrison R.G., "A wide-range electrometer voltmeter for atmospheric measurements in thunderstorms and disturbed meteorological conditions", Review of Scientific Instruments, 2002, vol 73, no 2, pp 482-483

${ }^{7}$ Harrison R.G., "A portable picoammeter for atmospheric electrical use", Institute of Physics Conference Series, 1995, vol 143, no 7, pp 223-226

${ }^{8}$ Harrison R.G and Aplin K.L., "Femtoampere current reference stable over atmospheric temperatures" , Review of Scientific Instruments, 2000, vol 71, no 8, pp 3231-3232
} 